\title{
COMÉRCIO AMBULANTE DE ALIMENTOS: CONDIÇÕES HIGIÊNICO-SANITÁRIAS NOS PONTOS DE VENDA NO MUNICÍPIO DE UMUARAMA, PARANÁ, BRASIL
}

\author{
Janaina Alves Magalhães ${ }^{1}$ \\ Simone dos Santos Carvalho ${ }^{2}$ \\ Regina Melquiades ${ }^{3}$ \\ Gilneia da Rosa ${ }^{4}$ \\ Luiz Sérgio Merlini ${ }^{5}$
}

MAGAlHÃES, J. A.; CARVALHO, S. dos S.; MELQUIADES, R.; ROSA, G. da; MERLINI, L. S. Comércio ambulante de alimentos: condições higiênico-sanitárias nos pontos de venda no município de Umuarama, Paraná, Brasil. Arq. Ciênc. Vet. Zool. UNIPAR, Umuarama, v. 19, n. 3, p. 147-152, jul./set. 2016.

RESUMO: A evolução no comércio de alimentos em vias públicas levou ao surgimento de um novo tipo de atividade econômica denominada comércio ambulante de alimentos ou street food, fazendo referência ao comércio de alimentos prontos e servidos. Denomina-se alimento servido, qualquer alimento ou bebida preparada e/ou vendida em ruas ou locais públicos, onde há etapas adicionais de preparo ou processamento. Este trabalho teve como objetivo traçar o perfil dos vendedores (idade, sexo, escolaridade) e analisar as condições higiênicas sanitárias do local do comércio ambulante de alimentos por meio da aplicação de check-list em 28 estabelecimentos de comércio ambulante de lanches no município de Umuarama, PR. Quanto ao perfil dos manipuladores de alimentos (vendedores ambulantes) 68\% eram do sexo masculino e $32 \%$ do sexo feminino. Em relação à faixa etária, verificou-se que 57\% apresentavam idade entre 36 e 50 anos, e $43 \%$ entre 18 e 35 anos, e nenhum acima de 51 anos. A formação escolar dos entrevistados variou entre primeira a quarta série com 18\%, até a oitava série $43 \%$ e segundo grau completo com 39\%. No que diz respeito ao tempo de serviço, $75 \%$ dos entrevistados atuavam na área a mais de seis anos, $14 \%$ até cinco anos e $11 \%$ até dois anos. Em relação às edificações e instalações, manipuladores e produção de alimentos, 63\% apresentam não conformidade com a legislação, demonstrando que se faz necessária uma maior fiscalização por parte da Vigilância Sanitária local, além da realização de programas de capacitação em boas práticas de manipulação para os manipuladores.

PALAVRAS-CHAVE: Alimentos de rua. Contaminação. Inspeção sanitária. Saúde única.

\section{STREET FOOD: HYGIENIC AND SANITARY CONDITIIONS IN OUTLETS IN THE CITY OF UMUARAMA, PARANÁ, BRAZIL}

\begin{abstract}
The evolution of food trade on the street has led to the rising of a new type of economic activity known as street food, which is the food ready to be served and eaten. Served food means any kind of food or beverage prepared and/ or sold in streets or public places where there are additional preparation or processing steps. This study aimed to analyze the hygienic and sanitary conditions of street food through the application of a checklist on 28 street trading locations in the city of Umuarama, PR. Regarding the profile of food handlers (street vendors), $68 \%$ are male and $32 \%$ are female. In terms of age, $57 \%$ were aged between 36 and 50 years old, while $43 \%$ were aged between 18 and 35 years, and none was older than 51. Schooling of the respondents ranged from $18 \%$ attending $1^{\text {st }}$ to $4^{\text {th }}$ year of elementary school, $43 \%$ attending the $8^{\text {th }}$ year of elementary school, and 39\% finishing high school. Regarding time on the job, $75 \%$ of the respondents worked in the area for more than six years, $14 \%$ up to five years, and 11\% up to two years. Regarding facilities, handlers and food production, $63 \%$ were non-compliant with the legislation, showing the need for a greater inspection by the local Health Surveillance agency, as well as holding training programs in good handling practices for food handlers.
\end{abstract}

KEYWORDS: Contamination. Sanitary inspection. Single health. Street food.

\section{COMERCIO DE ALIMENTOS CALLEJERO: CONDICIONES HIGIÉNICAS Y SANITARIAS EN PUESTOS DE VENTAS EN LA CIUDAD DE UMUARAMA, PARANÁ, BRASIL}

RESUMEN: La evolución del comercio de alimentos en vías públicas llevó a la aparición de un nuevo tipo de actividad económica, conocido como comercio de alimentos callejero o street food, haciendo referencia al comercio de alimentos preparados y servidos. Se llama alimento servido, cualquier alimento o bebida preparada y/o vendida en las calles o lugares públicos, donde hay etapas adicionales de preparo o procesamiento. Este estudio tuvo como objetivo buscar el perfil de los vendedores (edad, sexo, escolaridad) y analizar las condiciones higiénicas y sanitarias de locales de ventas de alimentos callejeros, a través de la aplicación de lista control en 28 establecimientos callejeros en la ciudad de Umuarama, PR. En cuanto

DOI: https://doi.org/10.25110/arqvet.v19i3.2016.6087

${ }^{1}$ Tecnóloga em alimentos, especialista em Vigilância Sanitária e Epidemiologia em Saúde. - janainaalvesmagalhaes@hotmail.com

${ }^{2}$ Tecnóloga em alimentos, especialista em Vigilância Sanitária e Epidemiologia em Saúde - mony umuarama@hotmail.com

${ }^{3}$ Biomédica, especialista em Vigilância Sanitária e Epidemiologia em Saúde - regina.melquiades@hotmail.com

${ }^{4}$ Acadêmica curso de Med. Veterinária da Universidade Paranaense - Unipar, bolsista PEBIC/CNPq

${ }^{5}$ Médico Veterinário, Mestre, Doutor do Programa de Pós-Graduação em Ciência Animal com ênfase em produtos Bioativos. 
al perfil de los manipuladores de alimentos (vendedores ambulantes), $68 \%$ son del sexo masculino y $32 \%$ del sexo femenino. Cuanto a la edad, se encontró que 57\% tenía entre 36 y 50 años, y 43\% entre 18 y 35 años, y no más de 51 años. La educación escolar de los encuestados osciló entre el $1^{\circ}$ y $4^{\circ}$ año de la primaria $18 \%$, hasta $8^{\circ}$ año de la primaria $43 \%$, la enseñanza secundaria completa 39\%. Con respecto al tiempo de trabajo, $75 \%$ de los encuestados trabajan en el área hace más de seis años, 14\% hasta cinco años y $11 \%$ hasta dos años. En relación a las edificaciones e instalaciones, manipuladores y producción de alimentos, $63 \%$ presentan incumplimiento con la legislación, demostrando que se hace necesaria una fiscalización mayor por parte de la Vigilancia Sanitaria local, además de realización de programas de capacitación en buenas prácticas de manipulación para los manipuladores.

PALABRAS CLAVE: Comida callejera. Contaminación. Salud única. Inspección sanitaria.

\section{Introdução}

O comércio de alimentos preparados e comercializados por vendedores ambulantes tem aumentado significativamente no Brasil, já que as pessoas estão trocando suas refeições caseiras pelas rápidas e práticas, que podem ser encontradas perto do seu local de trabalho ou estudo. Porém, essa mudança de hábito pode constituir um alto risco para a saúde dos consumidores, visto que as pessoas envolvidas nessa atividade geralmente não possuem um preparo para a manipulação adequada dos alimentos (PARISSENTI et al., 2013)

Entende-se por alimentos comercializados por ambulantes, alimentos e bebidas prontos para o consumo, preparados e/ou vendidos nas ruas e outros lugares públicos similares, para consumo imediato ou posterior, sem que haja, contudo, etapas adicionais de preparo ou processamento (CARMO, 2012).

As comidas vendidas nas ruas não têm garantia de inocuidade. Os pratos são montados na hora, com alimentos que se encontram expostos à contaminação ambiente (poeira, vento, sol) e na maioria das vezes armazenados em recipientes de plásticos sem qualquer refrigeração, sem contar com a infraestrutura inadequada (falta de água potável, rede de esgoto) e por vendedores que manipulam os alimentos desconhecendo as boas práticas de manipulação dos alimentos (MONTEIRO, 2015).

Além disso, em muitas cidades não há fiscalização, e, quando existe, geralmente não contempla as questões socioeconômicas do manipulador e seus conhecimentos sobre higiene e saúde, mas simplesmente a ocupação do espaço urbano e a posse de licenças (AKUTSU et al., 2015).

No caso de Umuarama, localizada no estado do Paraná, não existe, uma lei específica para vendedores ambulantes, existindo apenas uma seção na lei complementar $n^{\circ}$ 129 de 22 de dezembro de 2004, que dispõe sobre o código de postura do município, que rege algumas regras para esse tipo de comércio, porém como citado acima, não é aplicada corretamente (BRASIL, 2004).

A preocupação com a segurança alimentar vem crescendo nos últimos anos, gerando uma série de discussões entre organizações governamentais, instituições de ensino e indústrias alimentícias sobre programas que assegurem à população produtos que não sejam prejudiciais à saúde. Essa questão, que a princípio envolvia basicamente a disponibilidade e possibilidade de acesso da população ao alimento, está sendo discutida também em função dos riscos causados por esses mesmos alimentos (MALLON; BORTOLOZO, 2004).

A ocorrência de Doenças Transmitidas por Alimentos (DTA) vem aumentando de modo significativo em nível mundial. Vários são os fatores que contribuem para a emergência dessas doenças, entre os quais se destacam: o crescente aumento das populações; a existência de grupos populacionais vulneráveis ou mais expostos; o processo de urbanização desordenado e a necessidade de produção de alimentos em grande escala. Contribui ainda, o deficiente controle dos órgãos públicos e privados no tocante a qualidade dos alimentos ofertados às populações (BRASIL, 2012).

As DTA's podem ser subdivididas em intoxicações alimentares, quando causadas pela ingestão de toxinas pré-formadas, sendo esta toxina formada durante a proliferação do microrganismo patogênico no alimento, e infecção, sendo causada pela ingestão de alimentos contendo células viáveis dos microrganismos patogênicos, onde eles se aderem à mucosa do intestino humano, se proliferando e se colonizando. Podendo ocorrer ainda, a invasão da mucosa do intestino e a proliferação de toxinas que alteram o funcionamento das células do trato gastrointestinal (FRANCO; LANDGRAF, 2008).

Para evitar a ocorrência dessas DTA, há a necessidade da implantação dos programas de qualidade, pois estes refletem diretamente na qualidade do produto final, visando a segurança do alimento e a saúde do consumidor, e dentro desses programas temos as BPF (Boas Práticas de Fabricação).

De acordo com a RDC n²16 de 15 de setembro de 2004, as Boas Práticas de Fabricação (BPF), estabelecem procedimentos para serviços de alimentação a fim de garantir as condições higiênico-sanitárias do alimento preparado e a conformidade dos alimentos com a legislação sanitária (BRASIL, 2004).

No manual de BPF, para que se produzam alimentos seguros e de qualidade, são considerados fatores como, a qualidade da matéria-prima utilizada, as condições estruturais e higiênicas dos equipamentos e instalações, as técnicas empregadas para manipulação dos alimentos e ainda a saúde dos manipuladores (COLOMBO; OLIVEIRA; SILVA, 2009).

Para que a implantação das BPF seja eficaz, é necessário ainda que hajam treinamentos com os colaboradores, para conscientização e aplicação, garantindo assim, a eliminação da inadequações existentes e a produção de um alimento seguro (COLOMBO; OLIVEIRA; SILVA, 2009).

Este trabalho teve como objetivo traçar o perfil dos vendedores (idade, sexo, escolaridade) e analisar as condições higiênicas sanitárias do local do comércio ambulante de alimentos por meio da aplicação de check-list em 28 estabelecimentos de comércio ambulante de lanches no município de Umuarama, PR. 


\section{Material e Métodos}

Trata-se de uma pesquisa de campo, observacional de aspecto qualitativo, onde foram avaliados 28 pontos de comercialização de alimentos servidos nas ruas na cidade de Umuarama-PR, no mês de abril de 2015.

A cidade de Umuarama está localizada na região noroeste do estado do Paraná (latitude $23^{\circ} 47^{\prime} 55$ Sul e longitude $53^{\circ} 18^{\prime} 48$ Oeste) e possui 100.676 habitantes (IBGE, 2012), possuindo miscigenação populacional de várias etnias, com diversos hábitos comportamentais e culturais.

Os pontos de vendas analisados foram escolhidos como sendo os com maior movimento de clientes, e a pesquisa foi realizada em dias de grande movimento (sexta-feira e sábado a noite). A razão dessa escolha foi a de analisar as condições em que estes alimentos estavam sendo preparados, se estava de acordo com a legislação, e se é dada a atenção necessária, pelo órgão fiscal local (Vigilância Sanitária), a estes estabelecimentos.

Os pontos de vendas foram avaliados por meio de análise visual e utilizou-se também, um check-list elaborado de acordo com a Resolução n ${ }^{\circ} 275$ (BRASIL, 2002), para verificação das condições higiênico-sanitárias, referentes as edificações e instalações, manipuladores e produção dos alimentos.

Foi aplicado um check-list com perguntas sobre o perfil do vendedor ambulante, faixa etária e escolaridade. Para isso, essa pesquisa foi previamente aprovada no Comitê de Ética em Pesquisa Envolvendo Seres Humanos $(\mathrm{CEPEH})$ da Universidade Paranaense, sob número CAAE: 47726315.5.0000.0106.

Para determinação dos resultados, utilizaram-se valores numéricos, onde foi calculado em percentual cada item analisado de conformidade e não conformidade. Após obtenção dos dados, os mesmos foram apresentados em forma de figuras e quadros.

\section{Resultados e Discussão}

O perfil dos manipuladores de alimentos (vendedores ambulantes) da cidade de Umuarama-PR mostrou que $68 \%$ são do sexo masculino e $32 \%$ do sexo feminino (Figura 1). Em relação à faixa etária dos entrevistados, verificou-se que $57 \%$ apresentavam idade entre 36 e 50 anos, e $43 \%$ entre 18 e 35 anos, e nenhum acima de 51 anos (Figura 2).

Figura 1: Perfil dos 28 vendedores ambulantes de alimentos. Umuarama - PR. 2015.

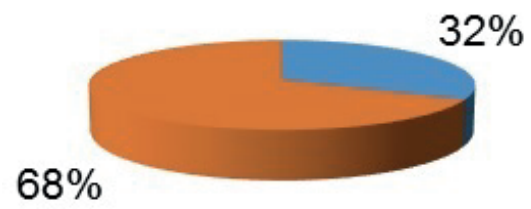

\section{-Feminino Masculino}

Observando-se os dados relativos ao perfil dos vendedores, percebeu-se que a maioria dos vendedores era do sexo masculino, contrapondo-se à tendência da América Latina, onde a venda de comida de rua é exercida principalmente por mulheres (HANASHIRO et al., 2013). Cardoso et al. (2005) obtiveram resultados semelhantes, que 68\% dos comerciantes eram do sexo masculino, em pesquisa realizada na cidade de Salvador - BA.

Figura 2: Faixa etária dos 28 vendedores ambulantes de alimentos. Umuarama- PR. 2015.

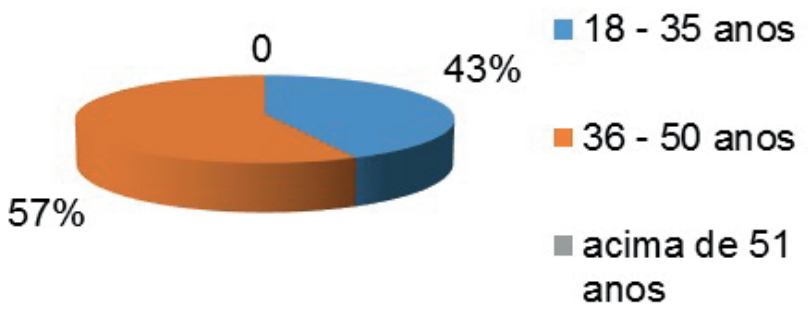

A média de idade entre os entrevistados foi de 3650 anos e amplitude de 18 a 51 anos, indicando a inserção de indivíduos da faixa etária economicamente ativa (Figura 2).

A formação escolar dos entrevistados variou entre primeira a quarta série com $18 \%$, até a oitava série com $43 \%$ e segundo grau completo com 39\% (Figura 3). Em estudos realizados por Abreu, Medeiros e Santos (2011), os autores demonstraram resultados semelhantes, em que, o grau de escolaridade entre os manipuladores era de $25 \%$ com ensino fundamental e $66,66 \%$ com ensino médio. Esta realidade revela uma característica importante, pois, alguns conhecimentos em relação à riscos e contaminação alimentar são adquiridos no ensino fundamental e médio.

Em estudo realizado por Silva et al. (2005), evidenciou-se a relação direta entre a formação dos vendedores e a condição higiênica sanitária no manuseio de alimentos e produtos comercializados, uma vez que o nível educacional configura um dos principais determinantes do risco à saúde associado à comida de rua.

Figura 3: Nível de escolaridade dos 28 vendedores ambulantes de alimentos. Umuarama- PR. 2015.

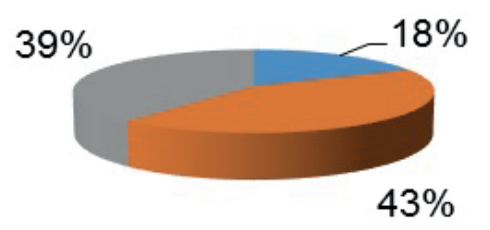

$1^{\mathrm{a}}$ a $4^{\mathrm{a}}$ série

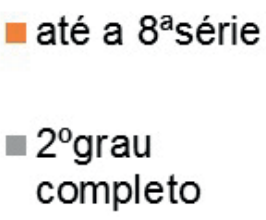

No que diz respeito ao tempo de serviço, $75 \%$ dos entrevistados atuam na área há mais de seis anos, 14\% até cinco anos e $11 \%$ até dois anos (Figura 4). De acordo com Camargo, Ferreira e Alves (2013), o complemento de renda foi apontado por $40 \%$ dos entrevistados como principal motivo para tornarem-se trabalhadores ambulantes. Tal fato revela, o motivo pelo qual esses vendedores ambulantes estão neste ramo há tanto tempo.

O tempo de serviço desses ambulantes poderia ser 
um fator positivo na melhoria constante dos serviços prestados, levando a uma diminuição dos riscos de contaminação alimentar durante a comercialização dos alimentos. Um dos problemas encontrados pelos ambulantes, segundo levantamento de dados, é a falta de qualificação profissional, o que de certa forma se reflete na atual situação do local.

Figura 4: Tempo de serviço na área de alimentos dos 28 vendedores ambulantes. Umuarama- PR. 2015.

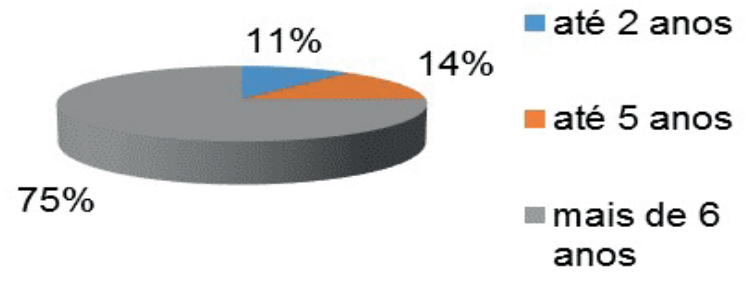

No Quadro 1, estão apresentadas as conformidades encontradas nas edificações e instalações, onde verificou-se que $50 \%$ dos locais avaliados, não possuíam local adequado com água corrente para lavagem das mãos e utensílios, porém, de acordo com a RDC $n^{\circ} 275$, é obrigatório a existência de lavatórios na área de manipulação com água corrente, dotados preferencialmente de torneira com acionamento automático (BRASIL, 2002).

Constatou-se um maior percentual de não conformidades no item presença de insetos no local (79\%), seguido de procedimento de higienização do local (68\%). As falhas nos procedimentos de higienização de equipamentos e utensílios permitem que os resíduos aderidos aos equipamentos e superfícies se transformem em potencial fonte de contaminação cruzada (CHESCA et al., 2003).

A limpeza de bancadas e superfícies, em 57\% dos estabelecimentos, era realizada de maneira adequada. E em relação à existência de acúmulo de lixos, $65 \%$ estão em conformidade.

Quadro 1: Conformidades de edificações e instalações dos comerciantes ambulantes de lanches ( $\mathrm{n}=28)$ do município de Umuarama - PR. 2015.

\begin{tabular}{|l|c|c|}
\hline \multicolumn{1}{|c|}{ Edificações e Instalações } & Conformidade (\%) & Não Conformidade (\%) \\
\hline Lavatório com água corrente para lavagem das mãos e utensílios & 50 & 50 \\
\hline Procedimento de higienização do local & 32 & 68 \\
\hline Bancadas e superfícies estão limpas & 57 & 43 \\
\hline $\begin{array}{l}\text { Existe focos de poeira " poluição", e acúmulo de lixo nas proximidades } \\
\text { do local }\end{array}$ & 65 & 35 \\
\hline Presença de insetos no local & 21 & 79 \\
\hline
\end{tabular}

O quadro 2 apresenta os hábitos dos manipuladores. Ao iniciar a pesquisa, a maioria dos vendedores ambulantes demonstrou resistência para compreender a finalidade do estudo realizado, os quais relataram que os mesmos recebiam orientações quanto aos riscos de contaminação $(71 \%)$, po- rém, conforme observado durante a avaliação visual, no momento da manipulação, isso não foi constatado. Os mesmos reclamaram ainda, que não recebem apoio do município para adequação dos serviços.

Quadro 2: Conformidades de manipuladores dos comerciantes ambulantes de lanches ( $\mathrm{n}=28)$ do município de Umuarama - PR. 2015.

\begin{tabular}{|l|c|c|}
\hline \multicolumn{1}{|c|}{ Manipuladores } & Conformidade (\%) & Não Conformidade (\%) \\
\hline $\begin{array}{l}\text { Os manipuladores recebem orientações quanto aos riscos de } \\
\text { contaminação }\end{array}$ & 71 & 29 \\
\hline Realizam o procedimento correto de lavagem das mãos & 11 & 89 \\
\hline Utilizam papel toalha para secagem das mãos & 21 & 79 \\
\hline Os manipuladores, estão em bom estado de saúde & 100 & 0 \\
\hline Fazem exames periódicos & 36 & 64 \\
\hline Utilizam uniformes ou avental & 15 & 85 \\
\hline Mantêm unhas curtas, limpas e sem esmalte & 68 & 32 \\
\hline Utilizam adornos (anéis, brincos, etc.) & 79 & 21 \\
\hline Utilizam luvas & 39 & 61 \\
\hline Utilizam máscara & 0 & 100 \\
\hline Utilizam touca & 36 & 64 \\
\hline Quem manipula os lanches manipula dinheiro & 46 & 54 \\
\hline
\end{tabular}

Quanto ao procedimento de lavagem das mãos, $89 \%$ estavam em não conformidade, sendo que $79 \%$ não utilizavam papel toalha para secagem das mãos. Isso pode ser considerado um problema já que as mãos dos manipuladores de alimentos podem constituir fonte potencial de patógenos em serviços de alimentação (ABREU; MEDEIROS; SANTOS, 2011).

Todos os ambulantes apresentaram bom estado de 
saúde, no entanto, apenas 36\%, afirmaram realizar exames periódicos.

Em relação à utilização de uniformes, apenas $15 \%$ estavam em adequação com as normas estabelecidas. A maioria dos vendedores ambulantes não usava avental ou jaleco, utilizando como uniformes camisetas coloridas.

$\mathrm{Na}$ avaliação do asseio pessoal, 68\% apresentavam unhas curtas e sem esmalte e $79 \%$ não utilizavam adornos. Cabe destacar, que tais resultados são relevantes, uma vez que as unhas e os adornos são considerados grandes veículos de contaminação alimentar (BRASIL, 2004).

Em relação ao uso das luvas, 61\% não utilizavam; $100 \%$ dos vendedores ambulantes não utilizavam máscaras, e $64 \%$ não utilizavam toucas, sendo que a maioria destes fazia o uso de bonés e como consequência os cabelos não ficam protegidos.

Ainda se tratando dos hábitos higiênicos, $46 \%$ dos manipuladores não manipulam dinheiro, considerando-se que o preparo de alimentos e o manejo de dinheiro pela mesma pessoa acarretam em fator de risco para segurança alimentar (BRASIL, 2004).

No quadro 3, estão apresentadas as conformidades e não conformidades referentes à produção de alimentos.

Dos 28 entrevistados, $71 \%$ realizavam o armazenamento corretos das matérias primas perecíveis, destacando-se que a qualidade do produto final depende também das condições de armazenamento das matérias primas, garantindo assim um alimento seguro.
As bisnagas para catchup, mostarda, maionese, molho de pimenta ainda são muito utilizadas pelos vendedores ambulantes, constatando-se que $81 \%$ ainda realizam essa prática, sendo que a maioria dos locais deixava as bisnagas expostas à temperatura ambiente antes e após o consumo dos clientes.

Além disso, deve-se levar em conta que o manuseio das bisnagas é realizado por todos os clientes e nem todos realizam a higienização das mãos, aumentando o risco de contaminação. Outro fator importante é a validade, pois, na maioria das vezes, as bisnagas são reabastecidas sem controle de quanto tempo o produto está ali.

A avaliação da qualidade higiênico-sanitária dos pontos de vendas, incluindo a identificação do asseio corporal dos manipuladores, contribui sobremaneira para a garantia da qualidade microbiológica dos alimentos de rua (WHO, 2007). Diversos investigadores têm demonstrado que a falta de asseio corporal dos manipuladores é um dos principais focos de contaminação dos alimentos comercializados por ambulantes (MUYANJA et al., 2011). No entanto, uma série de outros fatores também pode comprometer a qualidade dos alimentos de rua, ou seja, temperatura e local de acondicionamento inapropriado (KIM et al., 2013); exposição e manuseio sem a devida proteção (MUYANJA et al., 2011); e condições inadequadas do meio ambiente, com exposição dos alimentos a insetos e animais domésticos (VAN'T RIET et al., 2003).

Quadro 3: Conformidades em relação à produção de alimentos dos comerciantes ambulantes de lanches. Umuarama - PR.

\begin{tabular}{|l|c|c|}
\hline \multicolumn{1}{|c|}{ Produção de Alimentos } & Conformidade (\%) & Não Conformidade (\%) \\
\hline As matérias-primas perecíveis ficam refrigeradas & 71 & 29 \\
\hline Condimentos são em sachê? & 19 & 81 \\
\hline
\end{tabular}

\section{Conclusão}

As condições higiênicas sanitárias refletiram ações de não conformidades das edificações e instalações, manipuladores e produção de alimentos. Além disso, verificou-se que a falha na fiscalização do município, contribui para que essas não conformidades aconteçam, sendo que foi observado pelos próprios ambulantes que falta apoio do município, e que há ainda, necessidade de capacitações e treinamento para que assim possam promover mudanças de hábitos entre eles.

Campanhas educativas, também deveriam ser realizadas, pois, deste modo, contribuiria para melhorar a qualidade e segurança dos produtos comercializados.

\section{Referências}

ABREU, E. S.; MEDEIROS, F. S.; SANTOS, D. A. Análise microbiológica de mãos de manipuladores de alimentos do município de Santo André. Revista Univap, São José dos Campos-SP, v. 17, n. 30, 2011.

AKUTSU, R. C. et al. Adequação das boas práticas de fabricação em serviços de alimentação. Revista de Nutrição, Campinas, v. 18, n. 3, p. 419-427, 2015.

BRASIL. Resolução RDC n 275, de 21 de outubro de 2002. Dispõe sobre Regulamento Técnico de Procedimentos Operacionais Padronizados e Aplicados aos Estabelecimentos/Industrializadores de Alimentos e a Lista de Verificação das Boas Práticas de Fabricação em Estabelecimentos Produtores/Industrializadores de Alimentos. Diário Oficial da União, Brasília, 6 de novembro de 2002.

BRASIL. Ministério da Saúde. Agência Nacional de Vigilância Sanitária. Resolução RDC n²16, de 15 de setembro de 2004. Dispõe sobre Regulamentos Técnicos de Boas Práticas para Serviços de Alimentação. Diário Oficial da União, Brasília, 16 de setembro de 2004.

BRASIL. Ministério da Saúde. Secretaria de Vigilância em Saúde. Departamento de Vigilância Epidemiológica. Manual integrado de vigilância, prevenção e controle de doenças transmitidas por alimentos. Ministério da Saúde, Secretaria de Vigilância em Saúde, Departamento de Vigilância Epidemiológica. - Brasília: Editora do Ministério da Saúde, 2010.

CAMARGO, A.; FERREIRA, T. A. P. C.; ALVES, V. F. Condições higiênico-sanitárias de preparo da comida de rua comercializada por ambulantes em Itumbiara-Goiás.

Revista de Biotecnologia \& Ciência. Goiânia, v. 2, n. 2, p 
117-131, 2013.

CARDOSO, R. C. V. et al. Comida de rua: desvendando o mundo do trabalho e a contribuição social e econômica da atividade em Salvador-Ba. Conjuntura e Planejamento, Salvador: v. 7, n. 137, p. 45-51, 2005.

CARMO, G. M. I. Epidemiologia dos surtos de doenças transmitidas por alimentos no Brasil. Instituto de Saúde coletiva, Universidade Federal da Bahia, Salvador, BA. 2012, 40 f. Dissertação (Mestrado em Controle de Qualidade de Alimentos), Escola de Veterinária e Zootecnia da Universidade Federal de Goiás, Goiás, 2012.

CHESCA, A. C. et al. Equipamentos e utensílios de unidades de alimentação e nutrição: um risco constante de contaminação das refeições. Revista Higiene Alimentar, Mirandópolis, v. 17, n. 114-115, p. 20-23, 2003.

COLOMBO, M.; OLIVEIRA, K. M. P.; SILVA, D. L. D. Conhecimento das merendeiras de Santa Fé, PR, sobre higiene e boas práticas de fabricação na produção de alimentos. Revista Higiene Alimentar, Mirandópolis, v. 23, n. 170-171, p. 39-46, 2009.

FRANCO, B. D. G. de M.; LANDGRAF, M.

Microrganismos patogênicos de importância em alimentos. In: Microbiologia de Alimentos. São Paulo. Editora Atheneu, 2008.

IBGE - Instituto Brasileiro de Geografia e Estatística. Cidades, 2012. Disponível em: <http://www.ibge.gov.br/ cidadesat/topwindow.htm?1>. Acesso em: 18 ago. 2015.

KIM, S. A. et al. Temperature increase of foods in car trunk and the potential hazard for microbial growth. Food Control, v. 29, p. 66-70, 2013.

HANASHIRO, A. et al. Microbiological quality of selected street foods from a restricted area of São Paulo city, Brazil. Food Control, v. 16, p. 439-444, 2013.

MALLON, C.; BORTOLOZO, E. A. F. Q. Alimentos comercializados por ambulantes: Uma questão de segurança alimentar. Revista UEPG. Ciência Biológica e Saúde, Ponta Grossa, v. 10, n. 3/4, p. 65-76, 2004.

MONTEIRO, M. A. M. Caracterização do comércio ambulante de alimentos em Belo Horizonte. Revista DEMETRA: Alimentação, Nutrição \& Saúde, Rio de Janeiro, v. 10, n. 1, p. 87-97, 2015.

MUYANJA, C. et al. Practices, knowledge and risk factors of street food vendors in Uganda. Food Control, v. 22,n.1, p. 1551-58, 2011.

PARISSENTI, A. C. et al. Avaliação microbiológica de cachorros-quentes comercializados por vendedores ambulantes na cidade de Videira, SC. Unoesc \& Ciência ACBS, Joaçaba, v. 4, n.1. 32-37, 2013.
SILVA, J. O. et al. Enteroparasitoses e onicomicoses em manipuladores de alimentos do município de Ribeirão Preto, SP, Brasil. Revista Brasileira de Epidemiologia, São Paulo, v. 8, n. 4, p. 71-77, 2005.

VAN 'T RIET, H. et al. Determinants of non-home-prepared food consumption in two low-income areas in Nairobi. Nutrition, v. 19, n. 2, p. 1006-12, 2003.

WHO - World Health Organization. Food safety documents. Food safety and foodborne illness. Fact sheet $\mathrm{N}^{\circ} 237$.

Reviewed March. 2007. Disponível em: http://www.who. int/foodsafety/publications/en/ Acesso em: 09/ago/ 2015.

Recebido em: 19.09.2016 Aceito em: 20.12.2016 